\title{
Avaliação das Propriedades Físicas e Funcionais dos Dispositivos de Insuflação de Cateteres de Angioplastia Submetidos a Processo para Reutilização
}

\author{
Márcia Flores de Casco', Maria Antonieta Moraes², Emiliane Nogueira de Souza², \\ José Casco Raudales², Eneida Rejane Rabelo
}

\section{RESUMO}

Introdução: A prática de reprocessamento, de reesterilização e de reutilização de artigos odonto-médico-hospitalares é ampla no País e no mundo, e tem como objetivo fundamental reduzir os custos dos procedimentos. Os dispositivos de insuflação de cateteres de angioplastia não fogem a essa regra de reutilização. A avaliação das características físicas e funcionais desses dispositivos após processamento para sua reutilização permanece pouco explorada na literatura. Objetivos: Avaliar se as características físicas e funcionais dos dispositivos de insuflação de cateteres de angioplastia, após os processos para a reutilização, são mantidas, bem como determinar a vida útil de tais dispositivos. Método: Estudo longitudinal contemporâneo. Incluíram-se dispositivos de insuflação de cateteres de angioplastia novos, utilizados no período de novembro de 2007 a março de 2008. Estudo realizado em hospital universitário, localizado em Porto Alegre, Rio Grande do Sul, Brasil. As avaliações foram realizadas em dois momentos: grupo $\mathrm{A}$, dispositivos avaliados imediatamente após o primeiro uso; e grupo B, dispositivos avaliados após usos subsequentes. Resultados: Dos 36 dispositivos estudados, $11(30,6 \%)$ foram desprezados logo após o primeiro uso por apresentarem problemas mecâni$\cos$ e funcionais graves. Dos dispositivos restantes, apenas $8(32 \%)$ no grupo A e $6(24 \%)$ no grupo B mantiveram sua funcionalidade integralmente preservada. Esses instrumentais apresentaram alterações significativas, ainda que de pequena magnitude, nas aferições a partir de 12 atm. A média de reutilização foi de $1,7 \pm 1,2$ vez. Conclusões: Demonstrou-se, neste estudo, que cerca de $30 \%$ dos dispo-

\section{ABSTRACT}

Evaluation of the Physical and Functional

Properties of Angioplasty Insufflation Devices Undergoing Reuse Processing

Background: Reuse of dental and medical devices is a worldwide common practice, and our country is not an exception; its aim is to reduce procedural costs. PTCA insufflation devices (or manometers) are frequently resterilized for clinical reuse purposes. Evaluations of their physical and mechanical properties are not described in current literature. Objective: Determine if the physical and functional characteristics of insufflation devices are maintained after reuse processing, as well as establish their life span. Methods: This was a prospective longitudinal study, carried out at university hospital in Porto Alegre, RS, Brazil. From November 2007 to March 2008, all new insufflation devices were included and separated in two different groups: Group A devices were evaluated immediately after their first use and Group B devices were evaluated after subsequent uses. Results: Of the 36 devices, $11(30.6 \%)$ were discarded after the first use due to severe mechanical or functional problems. Of the remaining devices, only $8(32 \%)$ in Group A and $6(24 \%)$ in Group B kept totally preserved functionality. These devices presented significant changes, even though they were mild, in measurements after $12 \mathrm{~atm}$. Mean reuse rate was $1.7 \pm 1.2$ times. Conclusions: Results showed that about $30 \%$ of PTCA insufflation devices were not in working conditions after the first use. Only a quarter of the remaining

Monografia do Curso de Especialização: Enfermagem em Cardiologia - Instituto de Cardiologia - Fundação Universitária de Cardiologia (IC-FUC).

1 Unidade de Hemodinâmica do Hospital de Clínicas de Porto Alegre - Porto Alegre, RS, Brasil.

2 Residência Integrada em Cardiologia do Instituto de Cardiologia do Rio Grande do Sul/Fundação Universitária de Cardiologia - Porto Alegre, RS, Brasil.

Correspondência: Eneida R. Rabelo. Av. Princesa Isabel, 370 -

Santana - Porto Alegre, RS, Brasil - CEP 90620-000

E-mail: rabelo@portoweb.com.br

Recebido em: 25/3/2009 • Aceito em: 18/6/2009 
sitivos de insuflação de cateteres de angioplastia se apresentaram inadequados após a primeira utilização. E somente um quarto dos remanescentes manteve suas características funcionais íntegras até o final de sua vida útil.

DESCRITORES: Angioplastia transluminal percutânea coronária. Equipamentos e provisões hospitalares. Reutilização de equipamento. devices maintained intact functional characteristics to the end of their life span.

DESCRIPTORS: Percutaneous transluminal coronary angioplasty. Equipment and hospital supplies. Equipment reuse.
0 reprocessamento de materiais tem sido amplamente discutido na literatura ${ }^{1,2}$. Muitos desses materiais, conforme a recomendação do fabricante, têm indicação de uso único; contudo, pelo seu alto custo, as instituições de saúde acabam optando pela reutilização após reprocessamento ${ }^{1-4}$.

Procedimentos cardíacos, como angioplastia coronária transluminal percutânea e implante de endopróteses, necessitam de diferentes dispositivos para sua realização. Dentre eles destaca-se o dispositivo de insuflação, o qual consiste em uma seringa graduada que será preenchida com $20 \mathrm{ml}$ de solução de água destilada misturados a $20 \mathrm{ml}$ de contraste. O cateterbalão é preenchido sob pressão com essa solução, por meio de um sistema hidráulico controlado por um êmbolo com formato de parafuso, e tanto o controle como os ajustes da pressão de insuflação (usualmente aferida em atmosferas) são realizados por meio de um manômetro analógico acoplado à seringa. Do ponto de vista terapêutico, é fundamental para o operador saber se a pressão que está sendo exercida é exatamente aquela desejada e adequada para aquele balão. Pressões mais altas ou mais baixas podem se traduzir em resultados subótimos ou até trágicos ao paciente. Dessa forma, se uma determinada informação é de extrema relevância, o operador deve gastar tempo suficiente para obtê-la de maneira inequívoca ${ }^{5}$.

O dispositivo de insuflação de cateteres de angioplastia é considerado um artigo médico semicrítico, por estar diretamente conectado ao sistema terapêutico invasivo, e não consta da lista de artigos proibidos de reprocessamento (RE no 2605/ANVISA 2006) ${ }^{6}$. Assim, conforme normatização nacional, esse dispositivo é passível de reprocessamento mediante aplicação de um protocolo validado. Os protocolos de reprocessamento devem garantir a qualidade do resultado e de todas as etapas do processo, incluindo avaliação de funcionalidade, esterilidade, rastreabilidade, condições de armazenamento e descarte dos produtos (RDC 156/06, RE 2605/06 e RE 2606/06 da ANVISA) ${ }^{6-8}$. A validação dos protocolos de reprocessamento tem como objetivo garantir que o produto médico reprocessado tenha desempenho e segurança compatíveis com sua finalidade.

A preocupação com o reuso desse dispositivo vem gerando muitos desafios para os profissionais que utilizam diariamente esses produtos. A avaliação da manutenção das propriedades desses dispositivos permanece pouco explorada na literatura. Considerando que a investigação sobre a funcionalidade e a eficiência desse dispositivo contribuiria para qualificar a assistência prestada aos pacientes, delineou-se um estudo longitudinal, cujo objetivo foi avaliar se as características físicas e funcionais dos dispositivos de insuflação de cateteres de angioplastia, após os processos para a reutilização, são mantidas, bem como determinar a vida útil de tais dispositivos.

\section{MÉTODO}

Estudo longitudinal contemporâneo. A pesquisa foi realizada em Unidade de Hemodinâmica (UHD) de hospital público e universitário em Porto Alegre, Rio Grande do Sul, Brasil. Nessa unidade são realizadas, em média, 60 intervenções por mês que requerem o uso dos dispositivos de insuflação.

Todo dispositivo de insuflação novo utilizado em procedimento intervencionista, no período de novembro de 2007 a março de 2008, foi incluído no estudo. Após seu uso, foi realizada uma primeira limpeza com água corrente para eliminar resíduos de sangue e o dispositivo foi avaliado conforme protocolo (Apêndice 1). Para determinar a integridade dos dispositivos foram realizadas duas avaliações: uma avaliação visual (a conferência da integridade do sistema hidráulico à procura de eventuais vazamentos) e outra avaliação funcional (verificação da precisão do mecanismo do relógio analógico por meio de manômetro digital) por protocolo de pesquisa elaborado para este estudo, registradas em instrumento de coleta de dados (Apêndice 2). As diferenças de pressões entre o valor obtido na avaliação funcional e o valor nominal do dispositivo, expresso em porcentual, foram obtidas por meio do delta P [(pressão aferida/pressão nominal x 100\%) -100]. Este estudo foi aprovado pelo Comitê de Ética da instituição.

\section{Análise estatística}

As variáveis contínuas foram expressas como médias \pm desvio padrão, e as diferenças entre as atmosferas em cada reuso foram comparadas por meio de modelos lineares mistos, com correção de Bonferroni para as comparações múltiplas e os testes $t$ não-pareados e qui-quadrado. Todos os testes foram realizados com 
Casco MF, et al. Avaliação das Propriedades Físicas e Funcionais dos Dispositivos de Insuflação de Cateteres de Angioplastia Submetidos a Processo para Reutilização. Rev Bras Cardiol Invas. 2009;17(2):227-33.

\section{APÊNDICE 1 \\ Protocolo de Avaliação}

\section{Avaliação das propriedades físicas e funcionais dos dispositivos de insuflação de cateteres de angioplastia submetidos ao processo para reutilização}

Para determinar a integridade dos dispositivos serão realizadas duas avaliações, uma avaliação visual (conferência da integridade do sistema hidráulico à procura de eventuais vazamentos) e outra funcional (avaliação da precisão do mecanismo do relógio analógico por meio de manômetro digital), por meio do seguinte protocolo:

1. Todo dispositivo novo (primeira utilização) deverá ser rastreado. Uma ficha especialmente elaborada para esse rastreamento será preenchida com os dados do dispositivo (marca, modelo, capacidade volumétrica e características do relógio analógico). Nessa mesma ficha, serão registrados os dados obtidos nas subsequentes avaliações.

2. Nessa primeira utilização e uma vez usado na intervenção coronária percutânea, o dispositivo será identificado por uma marca ou sinalização específica $(R x$, onde $R=$ rastreamento e $x$ = número de identificação do dispositivo) e será conferida a integridade do sistema hidráulico à procura de eventuais vazamentos.

3. A seguir será avaliada a precisão do mecanismo do relógio analógico por meio de manômetro padronizado criado para fins de rastreamento.

4. Serão aferidas as pressões de 6, 8, 10, 12, 14, 16, 18 e 20 atm, comparando as leituras do relógio analógico com as leituras do manômetro padronizado, verificando se existem inconsistências nas leituras e registrando-as na ficha respectiva.

5. Depois dessa avaliação, o dispositivo sofrerá uma primeira limpeza e será encaminhado para o procedimento de reprocessamento usual.

6. Após cada uso do dispositivo, os procedimentos relativos aos itens 2, 3 e 4 serão repetidos e uma nova marca (um ponto) será colocada. Isso será repetido até que o dispositivo apresente sinais evidentes de dano no sistema hidráulico, no relógio ou quando ocorrerem inconsistências nas leituras com o manômetro padronizado.

7. Todo dispositivo, depois de confirmada sua integridade (visual e funcional), será limpo e seco, e posteriormente será encaminhado à empresa prestadora do serviço de reprocessamento, a qual rotineiramente realiza teste microbiológico, documentado com o laudo respectivo que acompanha a devolução do produto ao setor correspondente.

\section{TABELA 1}

Características dos dispositivos avaliados

\begin{tabular}{|c|c|}
\hline Características & $n=36$ \\
\hline Dispositivo da mesma marca & $36(100 \%)$ \\
\hline Pressão nominal* máxima 25 atm & $36(100 \%)$ \\
\hline Insuflações por uso & $2,06 \pm 0,47$ \\
\hline Reuso por dispositivo & $1,7 \pm 1,2$ \\
\hline $\begin{array}{l}\text { Dispositivos desprezados } \\
\text { após primeiro uso }\end{array}$ & $11(30,6 \%)$ \\
\hline Dispositivos reusados & $25(69,4 \%)$ \\
\hline \multicolumn{2}{|c|}{$\begin{array}{l}\text { * Pressão nominal: pressão original do dispositivo indicada } \\
\text { por um algarismo no manômetro analógico. } \\
\text { atm }=\text { atmosferas ( } 1 \mathrm{~atm}=760 \mathrm{mmHg} \text {; } \mathrm{n}=\text { número de } \\
\text { dispositivos. }\end{array}$} \\
\hline
\end{tabular}

o software Statistical Package for Social Science (SPSS) versão 14. As diferenças entre as avaliações do processo de reutilização foram consideradas significativas quando $\mathrm{P} \leq 0,05$.

\section{RESULTADOS}

A amostra foi constituída por 36 dispositivos da mesma marca e modelo, com capacidade máxima de $25 \mathrm{~atm}$. A média de insuflações, em cada procedimento, foi de 2,06 \pm 0,47 vezes. Dos 36 dispositivos, 11 $(30,6 \%)$ foram descartados após o primeiro uso por apresentarem vazamentos ou alterações no manômetro, e os 25 restantes $(69,4 \%)$ foram reutilizados (Tabela 1 ).

As avaliações funcionais dos dispositivos incluídos foram realizadas em dois momentos: grupo $A$, dispositivos avaliados imediatamente após o primeiro uso; e grupo B, dispositivos avaliados após usos subsequentes. Dos 25 dispositivos do grupo A, apenas $8(32 \%)$ mantiveram suas características funcionais integralmente, ou seja, apenas esses dispositivos forneceram leituras acuradas até 20 atm. Nos outros 17 (68\%), houve manutenção parcial de suas funções. Na Tabela 2, podemos observar que 100\% dos 25 dispositivos mediram com acurácia as pressões até 12 atm; a partir desse limiar, houve redução progressiva do número de dispositivos que alcançaram as pressões nominais indicadas no manômetro. 


\section{APÊNDICE 2}

\section{Dados do dispositivo e do procedimento}

\section{a) Marca:}

b) Modelo:

c) Capacidade volumétrica: $\mathrm{ml}$

d) Capacidade do relógio analógico: atm

e) Tipo de procedimento realizado:
ACTP com balão ( )
Stent ( )
Ambos ( )

f) Número de insuflações do dispositivo no mesmo procedimento:

Data
Data

№ de vezes:

№ de vezes:

№ de vezes:

№ de vezes:

№ de vezes:

№ de vezes:

№ de vezes:

№ de vezes:

№ de vezes:

\begin{tabular}{|c|c|c|c|c|c|}
\hline Data de uso & \# uso & Vazamento seringa & Vazamento relógio & Vazamento mangueira & Descartado \\
\hline 11 & 1 & $\mathrm{~S}(\quad) \mathrm{N}(\quad)$ & $\mathrm{S}(\quad) \mathrm{N}(\quad)$ & $\mathrm{S}(\quad) \mathrm{N}(\quad)$ & $S(\quad) N()$ \\
\hline 1 & 2 & $\mathrm{~S}(\quad) \mathrm{N}(\quad)$ & $\mathrm{S}(\quad) \mathrm{N}(\quad)$ & $\mathrm{S}(\quad) \mathrm{N}(\quad)$ & $S(\quad) N(\quad)$ \\
\hline 11 & 3 & $\mathrm{~S}(\quad) \mathrm{N}(\quad)$ & $\mathrm{S}(\quad) \mathrm{N}(\quad)$ & $\mathrm{S}(\quad) \mathrm{N}(\quad)$ & $S(\quad) N(\quad)$ \\
\hline 11 & 4 & $\mathrm{~S}(\quad) \mathrm{N}(\quad)$ & $\mathrm{S}(\quad) \mathrm{N}(\quad)$ & $\mathrm{S}(\quad) \mathrm{N}(\quad)$ & $\mathrm{S}(\quad) \mathrm{N}(\quad)$ \\
\hline 11 & 5 & $\mathrm{~S}(\quad) \mathrm{N}(\quad)$ & $\mathrm{S}(\quad) \mathrm{N}(\quad)$ & $\mathrm{S}(\quad) \mathrm{N}(\quad)$ & $\mathrm{S}(\quad) \mathrm{N}(\quad)$ \\
\hline 11 & 6 & $\mathrm{~S}(\quad) \mathrm{N}(\quad)$ & $\mathrm{S}(\quad) \mathrm{N}(\quad)$ & $\mathrm{S}(\quad) \mathrm{N}(\quad)$ & $\mathrm{S}(\quad) \mathrm{N}(\quad)$ \\
\hline 11 & 7 & $\mathrm{~S}(\quad) \mathrm{N}(\quad)$ & $\mathrm{S}(\quad) \mathrm{N}(\quad)$ & $\mathrm{S}(\quad) \mathrm{N}(\quad)$ & $S(\quad) N(\quad)$ \\
\hline 1 & 8 & $\mathrm{~S}(\quad) \mathrm{N}(\quad)$ & $\mathrm{S}(\quad) \mathrm{N}(\quad)$ & $\mathrm{S}(\quad) \mathrm{N}(\quad)$ & $S(\quad) N()$ \\
\hline 11 & 9 & $\mathrm{~S}(\quad) \mathrm{N}(\quad)$ & $\mathrm{S}(\quad) \mathrm{N}(\quad)$ & $S(\quad) N(\quad)$ & $S(\quad) N(\quad)$ \\
\hline
\end{tabular}

TABELA 2

Avaliação funcional dos dispositivos testados imediatamente após o primeiro uso - Grupo A

\section{Pressão nominal atingida} pelo dispositivo

\begin{tabular}{lc}
\hline Pressão $6 \mathrm{~atm}$ & $25(100 \%)$ \\
Pressão $8 \mathrm{~atm}$ & $25(100 \%)$ \\
Pressão $10 \mathrm{~atm}$ & $25(100 \%)$ \\
Pressão $12 \mathrm{~atm}$ & $25(100 \%)$ \\
Pressão $14 \mathrm{~atm}$ & $24(96 \%)$ \\
Pressão $16 \mathrm{~atm}$ & $16(64 \%)$ \\
Pressão $18 \mathrm{~atm}$ & $13(52 \%)$ \\
Pressão $20 \mathrm{~atm}$ & $8(32 \%)$ \\
\hline
\end{tabular}

$\operatorname{atm}=\operatorname{atmosferas}(1 \mathrm{~atm}=760 \mathrm{mmHg})$.
25 dispositivos/ 25 utilizações

$25(100 \%)$

\%

$5(100 \%)$

$24(96 \%)$

$13(52 \%)$

$8(32 \%)$
Os 25 dispositivos considerados ainda funcionalmente adequados foram reusados numa média de 1,7 \pm 1,2 vez (1 a 6 reusos), totalizando 35 reutilizações e constituindo o grupo B (Tabela 3). De maneira similar, os dispositivos reutilizados mantiveram a acurácia até 12 atm, com perda progressiva do número de instrumentais com leituras confiáveis a partir desse limite.

Comparando as médias de pressões aferidas entre os grupos A e B, não houve diferença estatisticamente significante até 16 atm (Tabela 4); no entanto, as médias para valores superiores foram significativamente maiores no grupo $A(P<0,014$ e $P<0,001$, respectivamente). Não houve diferenças significativas entre os grupos no que se refere aos dispositivos que mantiveram suas propriedades funcionais integrais em todas as faixas de pressão testadas até 20 atm.

Ocorreram diferenças de pressões entre o valor obtido na avaliação funcional e o valor nominal do 
Casco MF, et al. Avaliação das Propriedades Físicas e Funcionais dos Dispositivos de Insuflação de Cateteres de Angioplastia Submetidos a Processo para Reutilização. Rev Bras Cardiol Invas. 2009;17(2):227-33.

dispositivo expresso em porcentual em ambos os grupos, mostrando que a pressão realmente exercida pelo dispositivo reutilizado é maior que a determinada pelo

TABELA 3

Avaliação funcional dos dispositivos testados em subsequência ao primeiro uso - Grupo B

\begin{tabular}{lc}
\hline $\begin{array}{l}\text { Pressão nominal atingida } \\
\text { pelo dispositivo }\end{array}$ & $\begin{array}{c}\text { 25 dispositivos/ } \\
\text { 35 reutilizações }\end{array}$ \\
\hline Pressão 6 atm & $35(100 \%)$ \\
Pressão $8 \mathrm{~atm}$ & $35(100 \%)$ \\
Pressão $10 \mathrm{~atm}$ & $35(100 \%)$ \\
Pressão $12 \mathrm{~atm}$ & $35(100 \%)$ \\
Pressão $14 \mathrm{~atm}$ & $28(80 \%)$ \\
Pressão $16 \mathrm{~atm}$ & $19(54,3 \%)$ \\
Pressão $18 \mathrm{~atm}$ & $16(45,7 \%)$ \\
Pressão $20 \mathrm{~atm}$ & $10(28,6 \%)$ \\
\hline atm = atmosferas $(1 \mathrm{~atm}=760 \mathrm{mmHg})$. \\
\hline
\end{tabular}

seu manômetro, ainda que de pequena magnitude, acima de determinados limiares. No grupo A essa diferença foi evidente a partir de 10 atm e no grupo B, a partir de $8 \mathrm{~atm}$ (Tabela 5).

\section{DISCUSSÃO}

No presente estudo, avaliou-se se as características físicas e funcionais de 36 dispositivos de insuflação de cateter de angioplastia foram mantidas após os processos para a reutilização, bem como seu tempo de vida útil. Logo após a primeira utilização, um terço dos dispositivos apresentou alterações visíveis em suas características (vazamentos/ruptura no extensor/manômetro sem atingir o ponto zero) e foram desprezados. Dos dispositivos restantes, apenas 8 (32\%) no grupo A e $6(24 \%)$ no grupo B mantiveram sua funcionalidade integralmente preservada. Os demais dispositivos apresentaram alterações a partir de 12 atm. A média de reutilização foi de $1,7 \pm 1,2$ vez. Do ponto de vista prático, as pressões nominais exigidas em procedimentos cardiológicos oscilam entre 10 atm e 14 atm; no entan-

TABELA 4

Análise funcional comparativa entre os grupos A e B

\begin{tabular}{|c|c|c|c|}
\hline $\begin{array}{l}\text { Pressão nominal atingida } \\
\text { pelo dispositivo }\end{array}$ & $\begin{array}{c}\text { Grupo A } \\
\text { (25 dispositivos/25 utilizações) }\end{array}$ & $\begin{array}{c}\text { Grupo B } \\
\text { (25 dispositivos/35 reutilizações) }\end{array}$ & $\mathbf{P}$ \\
\hline Pressão 6 atm (intervalo mínimo-máximo) & $6,14 \pm 0,38(5,62-6,96)$ & $6,10 \pm 0,39(5,42-7,16)$ & NS \\
\hline Pressão 8 atm (intervalo mínimo-máximo) & $8,06 \pm 0,17(7,72-8,4)$ & $8,16 \pm 0,34(7,68-9,05)$ & 0,074 \\
\hline Pressão 10 atm (intervalo mínimo-máximo) & $10,2 \pm 0,16(9,8-10,55)$ & $10,18 \pm 0,28(9,77-0,96)$ & NS \\
\hline Pressão 12 atm (intervalo mínimo-máximo) & $12,19 \pm 0,19(11,73-12,57)$ & $12,24 \pm 0,32(11,73-12,92)$ & NS \\
\hline Pressão 14 atm (intervalo mínimo-máximo) & $14,26 \pm 0,18(13,84-14,56)$ & $14,17 \pm 0,27(13,61-14,87)$ & NS \\
\hline Pressão 16 atm (intervalo mínimo-máximo) & $16,33 \pm 0,18(15,97-16,60)$ & $16,29 \pm 0,21(15,95-16,80)$ & NS \\
\hline Pressão 18 atm (intervalo mínimo-máximo) & $18,38 \pm 0,20(17,95-18,64)$ & $18,22 \pm 0,16(17,96-18,55)$ & 0,014 \\
\hline Pressão 20 atm (intervalo mínimo-máximo) & $20,49 \pm 0,13(20,35-20,65)$ & $20,17 \pm 0,23(19,75-20,53)$ & 0,001 \\
\hline
\end{tabular}

NS = não-significante.

TABELA 5

Comparação entre o valor aferido e o valor nominal do manômetro

\begin{tabular}{|c|c|c|c|c|}
\hline $\begin{array}{l}\text { Pressão nominal atingida } \\
\text { pelo dispositivo }\end{array}$ & $\begin{array}{c}\text { Grupo A } \\
\text { (25 dispositivos/25 utilizações) }\end{array}$ & $\mathbf{P}$ & $\begin{array}{c}\text { Grupo B } \\
\text { (25 dispositivos/35 reutilizações) }\end{array}$ & $\mathbf{P}$ \\
\hline Pressão 6 atm & $6,07(\Delta \mathrm{P} 1,13 \%)$ & NS & $6,10(\Delta \mathrm{P} 1,67 \%)$ & NS \\
\hline Pressão 8 atm & $8,07(\Delta \mathrm{P} \quad 0,85 \%)$ & 0,086 & $8,15(\Delta \mathrm{P} 1,90 \%)$ & 0,010 \\
\hline Pressão 10 atm & $10,17(\Delta \mathrm{P} 1,67 \%)$ & 0,0001 & $10,18(\Delta \mathrm{P} 1,76 \%)$ & 0,001 \\
\hline Pressão 12 atm & $12,24(\Delta \mathrm{P} 2,02 \%)$ & 0,0001 & $12,23(\Delta \mathrm{P} 1,93 \%)$ & 0,0001 \\
\hline Pressão 14 atm & $14,27(\Delta \mathrm{P} 1,96 \%)$ & 0,0001 & $14,17(\Delta \mathrm{P} 1,23 \%)$ & 0,002 \\
\hline Pressão 16 atm & $16,38(\Delta \mathrm{P} 2,37 \%)$ & 0,0001 & $16,29(\Delta \mathrm{P} 1,80 \%)$ & 0,0001 \\
\hline Pressão 18 atm & $18,44(\Delta \mathrm{P} 2,42 \%)$ & 0,0001 & $18,21(\Delta \mathrm{P} 1,17 \%)$ & 0,0001 \\
\hline Pressão 20 atm & $20,49(\Delta \mathrm{P} 2,44 \%)$ & 0,0001 & $20,17(\Delta \mathrm{P} 0,82 \%)$ & 0,048 \\
\hline
\end{tabular}

NS = não-significante. 
to, não é raro ser necessário o uso de pressões maiores para atingir os resultados desejados, isto é, $16 \mathrm{~atm}$ ou $20 \mathrm{~atm}^{9,10}$. Consequentemente, em caso de usarmos um dispositivo que não mantenha essa pressão, é preciso fazer a troca por outro. Isso significa custos e dificuldade na operacionalização do procedimento.

O reprocessamento de materiais e dos dispositivos de angioplastia é factível, desde que seja seguido um protocolo de forma detalhada: limpeza, enxágue, secagem, empacotamento, rotulagem, esterilização e armazenamento. Deve ser descartado todo produto que não apresente condições de limpeza e esterilização adequadas ou que tenha perdido suas características funcionais ${ }^{8}$. A avaliação funcional dos dispositivos de insuflação de angioplastia é um procedimento viável e necessário em todos os estabelecimentos de saúde que optarem pelo reprocessamento desse material.

Em estudo realizado por Amarante et al. ${ }^{4}$ para avaliar a frequência de reprocessamento de materiais em 240 instituições, 119 (50\%) responderam ao questionário e 116 (97\%) relataram reprocessamento de materiais utilizados em procedimentos hemodinâmicos. No entanto, apenas $22 \%$ seguem um protocolo de reprocessamento padronizado ${ }^{4}$.

No Brasil e em diversos países, o reprocessamento de materiais é uma prática rotineira, predominantemente por razões econômicas. Desde 2006, quando a ANVISA $^{8}$ decretou a necessidade de protocolos validados para reprocessamento de todo material que não constasse de sua lista de "material de uso único", as instituições de saúde passaram a se reunir e procurar formas de se adequar a essas exigências.

O desenvolvimento de cateteres altamente sofisticados em cardiologia elevou os custos e, consequentemente, o reprocessamento torna-se uma questão em constante avaliação. Poucos hospitais seguem de fato protocolos sistemáticos para limpeza, esterilização e controle de qualidade relacionados à reutilização de cateter. Procedimentos claros e rígidos para prevenir possíveis eventos clínicos aos pacientes, como risco de infecções, reações pirogênicas, toxicidade e contaminações, devem ser urgentemente estabelecidos ${ }^{11}$.

\section{Limitações do estudo}

Neste estudo identificamos algumas limitações, como a avaliação de dispositivos de um único fabricante; portanto, estes resultados não podem ser generalizados. Por não ser estéril o manômetro de controle utilizado neste estudo, os dispositivos não foram testados antes de sua primeira utilização, e sua precisão, quando novos, foi realizada visualmente, avaliando a posição do ponto zero no manômetro. Também podemos considerar o fato de que o estudo foi conduzido em um hospital público, universitário, em que a compra de produtos é realizada por meio de licitação anual, limitando a avaliação de dispositivos de dife- rentes fabricantes simultaneamente. Além disso, durante o seguimento do estudo foram extraviados dois dispositivos quando enviados para reesterilização em empresa externa, conforme rotina da instituição.

\section{CONCLUSÃO}

Demonstrou-se, neste estudo, que cerca de $30 \%$ dos dispositivos de insuflação de cateteres de angioplastia se mostraram inadequados após a primeira utilização. Dos remanescentes, somente um quarto dos dispositivos submetidos a reprocessamento manteve sua funcionalidade preservada até o final de sua vida útil. Estudos futuros, com diferentes marcas, são sugeridos para confirmarem estes resultados.

\section{AGRADECIMENTOS}

Ao Centro de Pesquisa Cardiovascular e ao Departamento de Engenharia e Robótica da Universidade Luterana do Brasil, pelo design, construção e fornecimento do manômetro padronizado.

\section{CONFLITO DE INTERESSES}

Os autores declararam inexistência de conflito de interesses.

\section{REFERÊNCIAS BIBLIOGRÁFICAS}

1. Cohoon BD. Reprocessing of single-use devices. AORN J. 2002;75(3):557-62.

2. Qian Z, Castañeda WR. Can labeled single-use devices be reused? An old question in the new era. J Vasc Interv Radiol. 2002;13(12):1183-6.

3. Denser CPAC, Lacerda RA. Reprocessamento e reutilização de material odonto-médico-hospitalar de uso único: busca de evidências pela revisão sistemática de literatura científica. Acta Paul Enferm. 2006;19(3):316-22.

4. Amarante JM, Toscano CM, Pearson ML, Roth $\mathrm{V}$, Jarvis WR, Levin AS. Reprocessing and reuse of single-use medical devices used during hemodynamic procedures in Brazil: a widespread and largely overlooked problem. Infect Control Hosp Epidemiol. 2008;29(9):854-8.

5. Bregagnollo EA, Carvalho FC, Bregagnollo IF, Hirata JS. Aspectos metodológicos relacionados aos sistemas manométricos utilizados em estudos hemodinâmicos. Rev Bras Cardiol Invas. 2007;15(4):421-31.

6. Agência Nacional de Vigilância Sanitária - ANVISA. Resolução - RE no 2605, de 11 de agosto de 2006. Estabelece a lista de produtos médicos enquadrados como de uso único proibidos de ser reprocessados. Brasília: ANVISA; 2006. Disponível em: http://e-legis.anvisa.gov.br/leisref/public/ showAct.php?id=23407\&word

7. Agência Nacional de Vigilância Sanitária - ANVISA. Resolução RDC no 156, de 11 de agosto de 2006. Dispõe sobre o registro, rotulagem e reprocessamento de produtos médicos, e da outras providências. Brasília: ANVISA; 2006. Disponível em: http://e-legis.anvisa.gov.br/leisref/public/ showAct.php?id=23594\&word

8. Agência Nacional de Vigilância Sanitária - ANVISA. Resolução - RE n 2606, de 11 de agosto de 2006. Dispõe sobre as diretrizes para elaboração, validação e implantação de protocolos 
Casco MF, et al. Avaliação das Propriedades Físicas e Funcionais dos Dispositivos de Insuflação de Cateteres de Angioplastia Submetidos a Processo para Reutilização. Rev Bras Cardiol Invas. 2009;17(2):227-33.

de reprocessamento de produtos médicos e dá outras providências. Brasília: ANVISA; 2006. Disponível em: http://www. anvisa.gov.br/legis/resol/2006/re/2606_06re.htm

9. Mehran R, Mintz GS, Popma JJ, Pichard AD, Satler LF, Kent KM, et al. Mechanisms and results of balloon angioplasty for the treatment of in-stent restenosis. Am J Cardiol. 1996;78(6):618-22.
10. de Gregorio J, Colombo A. Treatment strategies for long and calcified lesions. J Interv Cardiol. 1996;11:557-64.

11. Batista MA, Santos MA, Pivatelli FC, Lima ARS, Godoy MF. Eventos adversos e motivos de descarte relacionados ao reuso de produtos médicos hospitalares em angioplastia coronária. Braz J Cardiovasc Surg. 2006;21(3):328-33. 